\title{
Análisis de servicios ecosistémicos para la configuración de una infraestructura verde en el área metropolitana de Sevilla
}

\author{
Jesús Santiago Ramos ${ }^{1}$ | Claudia Hurtado Rodríguez ${ }^{2}$ \\ Recibido: 25-11-2020 | en su versión final: 23-04-2021
}

Resumen

\begin{abstract}
La noción de infraestructura verde se ha consolidado en los últimos años como una potente herramienta conceptual y metodológica para la mejora ambiental en ámbitos urbanos y metropolitanos. Dos de sus principios esenciales son, de un lado, la interconexión física y funcional de los espacios verdes y zonas naturales presentes en el territorio, y de otro, el fomento de los servicios ecosistémicos en el entorno urbano y periurbano. Ambos objetivos suponen una nueva perspectiva para la planificación de las grandes aglomeraciones urbanas, e invitan a repensar nuestra forma de entender y ordenar el espacio libre metropolitano. El presente trabajo se propone aportar elementos de reflexión sobre esta cuestión, tomando como base el análisis de la provisión de servicios ecosistémicos y la cartografía de la multifuncionalidad ambiental en el área metropolitana de Sevilla. El cálculo de un conjunto de indicadores para servicios de regulación, provisión, culturales y vinculados a la preservación de la biodiversidad, junto con la estimación de un índice sintético de multifuncionalidad para el espacio libre, permitirán valorar las potencialidades y limitaciones que ofrece el concepto de infraestructura verde para su aplicación a escala metropolitana.
\end{abstract}

Palabras clave: Aglomeración urbana; espacio libre; indicadores ambientales; multifuncionalidad del paisaje

Citación

Santiago Ramos, J. y Hurtado Rodríguez, C. (2021). Análisis de servicios ecosistémicos para la configuración de una infraestructura verde en el área metropolitana de Sevilla. ACE. Architecture, City and Environment, 16(46), $9884 . \quad$ DOl http://dx.doi.org/10.5821/ace.16.46.9884

\section{An Analysis of Ecosystem Services for the Design of a Green Infrastructure in the Metropolitan Area of Seville}

Abstract

\begin{abstract}
The notion of green infrastructure has been consolidated in recent years as a powerful conceptual and methodological tool for environmental improvement in urban and metropolitan areas. Two of its basic principles are, on the one hand, the physical and functional interconnection of green spaces and natural areas present in the territory, and on the other, the promotion of ecosystem services in the urban and peri-urban environment. Both objectives represent a new perspective for the planning of large urban agglomerations and invite us to rethink our way of understanding and planning the metropolitan open space. This paper aims to provide some elements for reflection on this subject, based on the analysis of the provision of ecosystem services and the mapping of environmental multifunctionality in the Seville metropolitan area. The calculation of a set of indicators for regulation, provision, cultural and nature conservation-related services, together with the estimation of a synthetic multifunctionality index for the metropolitan open space, will allow us to assess the potential and limitations of the green infrastructure concept regarding its application at a metropolitan scale.
\end{abstract}

Keywords: Urban agglomeration; open space; environmental indicators; landscape mutifunctionality

${ }^{1}$ Doctor en Geografía. Profesor Ayudante Doctor, Universidad Pablo de Olavide (ORCiD: 0000-0001-75727763, ResearcherID: L-1657-2017, Scopus Author ID: 57208751460), ${ }^{2}$ Licenciada en Ciencias Ambientales. M.Sc. Técnico de investigación, Universidad Pablo de Olavide (ORCiD: 0000-0002-8056-4695, ResearcherID: K-6490-2017, Scopus Author ID: 56742686400). Correo de contacto: jsanram@upo.es 


\section{Introducción: del concepto de espacio libre a la noción de infraestructura verde}

A lo largo de las últimas décadas, la irrupción del concepto de sostenibilidad y la creciente preocupación ante los importantes retos ambientales que afronta nuestra sociedad han cambiado paulatinamente la forma en que estudiamos, planificamos y gestionamos las ciudades. Hoy en día entendemos la ciudad como un sistema territorial y ecológico complejo, en el que los elementos naturales y artificiales que lo integran se hallan íntimamente interrelacionados. Mantener un equilibrio entre ambos componentes del espacio urbanizado, siguiendo los criterios y principios que aportan disciplinas como la ecología urbana (Bettini, 1998) o la ecología del paisaje (Forman, 2008), resulta imprescindible para garantizar unos niveles aceptables de calidad ambiental en el medio urbano y, a la vez, minimizar el impacto ecológico de la ciudad sobre el territorio circundante. Una adecuada ordenación de los espacios libres y los elementos naturales asociados al entorno urbanizado es esencial para cumplir con este propósito.

Esta evolución hacia una visión más ecológica del urbanismo se ha producido en paralelo a otro importante cambio que también ha incidido en nuestra forma de entender las ciudades: el cambio de escala experimentado por los procesos urbanos durante la segunda mitad del siglo XX. La suma de factores diversos, que abarcan desde el incremento progresivo de la población urbana al aumento constante de la movilidad o la consolidación de nuevas pautas de vida en la ciudad -residenciales, de ocio, etc.- ha devenido en un claro redimensionamiento del fenómeno urbano en todo el planeta. La ciudad del siglo XXI es, como resultado, una ciudad metropolitana (Hall, 1998), que ha desbordado de manera definitiva los límites de la ciudad tradicional para adquirir una naturaleza mucho más extensa y compleja, tanto en su dimensión física como funcional. Ante esta nueva realidad, la escala municipal cada vez resulta más limitada e insuficiente para un análisis, una planificación y una gestión eficientes de la ciudad. En consecuencia, se hace necesario adoptar un enfoque metropolitano capaz de abarcar el sistema urbano en toda su complejidad (Feria, 2004). Desde el punto de vista de la sostenibilidad, la escala metropolitana parece también la más adecuada para abordar el impacto del crecimiento urbano sobre el territorio circundante, así como para aprovechar los potenciales beneficios que aporta el entorno periurbano para la mejora de la calidad ambiental y de las condiciones de vida de los ciudadanos (Feria y Santiago, 2019).

En el contexto del urbanismo y la planificación territorial, los cambios de perspectiva y de escala que hemos señalado tienen un reflejo evidente en la evolución de la terminología utilizada para la ordenación del espacio libre. En un principio, el concepto de espacio libre se ha asociado fundamentalmente a la escala y la perspectiva propias del planeamiento municipal. Desde esta óptica, las dos características fundamentales del espacio libre -ya sea bajo esta denominación o bajo otras como espacio abierto, espacio verde, verde urbano, etc.- serían su carácter público y su finalidad recreativa (Rodríguez-Avial, 1982; Acosta y Burraco, 1998). A ello se podría añadir el carácter eminentemente ordenado y mantenido de la vegetación presente en estos espacios, en su mayor parte parques y zonas ajardinadas. La aparición de iniciativas de análisis y planificación territorial de escala metropolitana ha supuesto la superación progresiva de los rasgos mencionados, ampliándose considerablemente el alcance y la complejidad de lo que entendemos por espacio libre. Desde una óptica metropolitana, el espacio libre podría definirse como "el suelo no dedicado a usos urbanos o paraurbanos, es decir, el espacio mayoritariamente exento de construcciones sea de uso forestal, pastoral, agrícola o plenamente silvestre, con independencia del estatuto jurídico o del régimen de propiedad a que esté sometido" (Folch, 2003: pág. 281). La noción de espacio libre se asimilaría así a la de matriz territorial, es decir, a la fracción no urbanizada del territorio que acoge y da soporte a los usos urbanos y artificiales, a la par que alberga valores ambientales estratégicos para la ordenación del territorio (Mata y Olcina, 2010). Esta concepción se aproximaría asimismo a otra noción propia de

ACE, 16 (4.6) CC BY-ND 3.0 ES | UPC Barcelona, España | Análisis de servicios ecosistémicos para la configuración de una infraestructura verde en el área metropolitana de Sevilla. DOI: http://dx.doi.org/10.5821/ace.16.46.9884 
la planificación territorial, la de sistema físico-ambiental o conjunto de "localizaciones y áreas preferentes para la protección de los recursos naturales, la preservación del paisaje y el patrimonio histórico, la prevención de riesgos, y la prevención y corrección ambiental” (Bielza, 2008: pág. 285).

A medida que se ha ido consolidando la perspectiva sostenibilista, el concepto de espacio libre ha ido ganando nuevos atributos tanto en lo relativo a su dimensión estructural como a su vertiente funcional (Cruz, Oliveira y Santiago, 2017). En primer lugar, se ha reconocido su papel como recurso para la provisión de un amplio rango de funciones o servicios ecosistémicos (Bolund y Hunhammar, 1999; Feria y Santiago, 2009; Fariña y Naredo, 2010). En segundo lugar, se ha consolidado su carácter sistémico, subrayándose la necesidad de interconectar los espacios abiertos y áreas naturales a través de corredores verdes u otros elementos de conexión (Batlle, 2006; Rueda, 2012). En tercer lugar, se ha remarcado la necesidad de transitar desde una lógica puramente conservacionista centrada en la protección de los espacios más valiosos frente al crecimiento urbano- hacia un enfoque más estratégico, orientado a la puesta en valor del espacio libre como activo para el desarrollo territorial (Agencia Europea de Medio Ambiente, AEMA, 2011a). Por último, se ha enfatizado la importancia del medio agrícola como componente básico del sistema de espacios libres, al que aporta valores y funciones de índole no sólo productiva y económica, sino también social, ambiental y paisajística (Batlle, 2011; Pedrazzini, 2011, Yacamán, Ferrer y Mata, 2020). Estos cambios se acompañan además de un progresivo interés del urbanismo por la renaturalización de la ciudad a través del adecuado tratamiento de los equipamientos verdes (Alomar-Garau, 2021), así como de un incremento de la proporción de espacios libres en los nuevos desarrollos residenciales (García, 2019).

El concepto que mejor refleja y sintetiza actualmente este cambio de paradigma en relación con el espacio libre metropolitano es sin duda el de infraestructura verde. Se trata de un nuevo enfoque para la ordenación y conservación de los recursos territoriales, que nace como respuesta a la problemática ecológica y social asociada a los nuevos modelos de crecimiento urbano difuso, al consumo acelerado de suelo y a la progresiva fragmentación de las áreas naturales (Benedict y McMahon, 2002). Desde su nacimiento, el concepto ha experimentado una amplia acogida en los ámbitos académico e institucional. Así, se ha incorporado al acervo de la UE a través de la Comunicación de la Comisión Europea "Infraestructura verde: mejora del capital natural de Europa" (Comisión Europea, 2013), que requiere a los países miembros la identificación y protección de los elementos territoriales que deben integrar sus respectivas infraestructuras verdes. A ello se une la inclusión del concepto en acciones y programas comunitarios tales como el Séptimo Programa Marco de Medio Ambiente o la Estrategia de la Unión Europea para el Cambio Climático. En España, la noción de infraestructura verde se incorpora al ordenamiento jurídico a través de la Ley 33/2015, por la que se modifica la Ley 42/2007 del Patrimonio Natural y de la Biodiversidad, y se consolida con la aprobación en 2020 de la Estrategia Nacional de la Infraestructura Verde y de la Conectividad y Restauración Ecológica. La presencia de la noción de infraestructura verde en los instrumentos de planificación metropolitana en España es aún poco significativa (Rodríguez y Aguilera, 2016; Cruz, Oliveira y Santiago, 2017; Feria y Santiago, 2017), si bien la clara apuesta institucional por esta herramienta como un instrumento estratégico para el desarrollo territorial sostenible y la cohesión social (AEMA, 2011a; AEMA, 2014) hace más que previsible su rápida consolidación en el contexto de la planificación de las grandes áreas urbanas. Algunos ejemplos destacados de aplicación a escala local -como la Infraestructura Verde de Vitoria-Gasteiz (Centro de Estudios Ambientales, 2014)- y subregional -como el Plan de Acción Territorial de la Infraestructura Verde del Litoral (PATIVEL) de la Comunidad Valenciana (Vera, Olcina y Sainz, 2019)- apuntan claramente en esta dirección.

Desde la perspectiva de la ordenación territorial, dos de los rasgos más relevantes que definen la noción de infraestructura verde son la conectividad y la multifuncionalidad (AEMA, 2011a; Feria y Santiago, 2017). En lo que respecta al primero de estos aspectos, la infraestructura verde metropolitana buscaría alcanzar un alto nivel de conectividad entre los espacios libres y áreas

ACE, 16 (4.6) CC BY-ND 3.0 ES | UPC Barcelona, España | Análisis de servicios ecosistémicos para la configuración 3 de una infraestructura verde en el área metropolitana de Sevilla. DOI: http://dx.doi.org/10.5821/ace.16.46.9884 
naturales, a fin de favorecer la conservación de los ecosistemas y contrarrestar los efectos que la fragmentación del paisaje y el aislamiento de los hábitats naturales tienen sobre la biodiversidad (Forman, 2008). Así mismo, desde la perspectiva del uso público, la interconexión de los espacios libres a través de elementos como vías verdes, corredores urbanos o carriles bici fomenta la movilidad sostenible en el entorno urbano y periurbano y facilita el acceso de los ciudadanos a los espacios de ocio al aire libre (Santiago, 2008).

En cuanto al segundo rasgo, la multifuncionalidad, el objetivo de una infraestructura verde metropolitana sería maximizar la suma total de servicios ecosistémicos que los ciudadanos pueden obtener del espacio libre. El concepto de servicio ecosistémico puede definirse como «las condiciones y procesos a través de los cuales los ecosistemas naturales y las especies que los integran sostienen y satisfacen las necesidades de la vida humana» (Daily, 1997); o, de forma más directa, "el beneficio que las personas obtienen de los ecosistemas» (Hassan et al., 2005). En el contexto metropolitano, estos servicios abarcan desde la reducción de la contaminación atmosférica (Manes et al., 2014) y la disminución de los impactos sobre el medio hidrológico (Lewellyn et al., 2016) a la mitigación de la isla de calor urbano (Farrugia et al., 2013) y la lucha contra el cambio climático (Momm-Schult et al., 2013). Asimismo, la infraestructura verde juega un papel relevante en relación con la producción de alimentos y la seguridad alimentaria (Magoni y Colucci, 2015) y la puesta en valor del patrimonio territorial local (Comisión Europea, 2013). Por último, la infraestructura verde atiende también a la dimensión social de la sostenibilidad, aportando beneficios intangibles de orden estético, cultural y recreativo y contribuyendo a generar un ambiente saludable para los ciudadanos (Tzoulas et al., 2007).

El objetivo de potenciar al máximo la multifuncionalidad del espacio libre metropolitano supone un reto evidente para cualquier iniciativa de planificación. Algunas de las funciones señaladas, como el fomento del uso público-recreativo y la conservación de los hábitats naturales a través de la mejora de su conectividad ecológica, responden claramente a un planteamiento en red; en este sentido, la infraestructura verde se configuraría como un conjunto de nodos o espacios clave articulados en red por medio de un conjunto de corredores o elementos lineales de conexión, ya sean de uso público o ecológicos. Sin embargo, muchas otras funciones de la infraestructura verde tendrían un encaje más difícil en este modelo reticular, al estar estrechamente ligadas a la matriz territorial y no tanto a un conjunto de espacios discretos; tal es el caso de la mayoría de las funciones productivas y de regulación ambiental que se han señalado. Articular unas y otras funciones, buscando sinergias y evitando posibles conflictos entre espacios y recursos territoriales de muy diferente naturaleza y vocación, es sin duda una tarea compleja.

El presente trabajo pretende aportar elementos de reflexión acerca de la configuración espacial óptima que debería asumir una infraestructura verde metropolitana de carácter multifuncional. Para ello, se toma como caso de estudio el área metropolitana de Sevilla, un territorio que por su diversidad, complejidad y riqueza paisajística ofrece importantes potencialidades a este respecto. Para el análisis de este ámbito, se ha tomado como referencia de base la propuesta metodológica de Maes, Paracchini y Zulian (2011) para el cálculo de indicadores espaciales de servicios ecosistémicos. Se han seleccionado indicadores relativos a tres servicios de regulación (mitigación del cambio climático, mejora de la calidad del aire y regulación hidrológica), dos servicios de provisión (producción agrícola y forestal) y un servicio cultural (uso público-recreativo). Se ha considerado asimismo como funcionalidad adicional la protección de los hábitats de mayor interés natural, una de las funciones más frecuentemente asignadas en el contexto de la planificación territorial a los sistemas de espacios libres metropolitanos y que suele jugar además un papel central en la configuración espacial de los mismos (Cruz, Oliveira y Santiago, 2017). En su conjunto, se ha buscado reflejar las facetas funcionales del territorio metropolitano que presentan un mayor interés para la conformación de una infraestructura verde.

ACE, 16 (4.6) CC BY-ND 3.0 ES | UPC Barcelona, España | Análisis de servicios ecosistémicos para la configuración de una infraestructura verde en el área metropolitana de Sevilla. DOI: http://dx.doi.org/10.5821/ace.16.46.9884 
Los indicadores se han clasificado en dos bloques: un primer grupo relativo a las funciones asociadas al concepto de red -funciones de conservación de hábitats de interés natural y de uso públicorecreativo- y un segundo grupo de funciones ligadas a la matriz territorial -funciones de regulación ambiental y de provisión. Esta categorización, orientada a facilitar el análisis y discusión de los resultados, no responde tanto a una visión jerárquica de los diferentes servicios ecosistémicos considerados, como a un enfoque horizontal estrechamente vinculado a la noción de multifuncionalidad del paisaje (De Groot y Hein, 2007), que resulta fácilmente extrapolable a la práctica de la ordenación territorial.

Los indicadores se han representado cartográficamente a fin de mostrar la distribución espacial de las diversas funciones en el territorio metropolitano. Finalmente, se ha obtenido una cartografía sintética de la multifuncionalidad del paisaje metropolitano a partir de la superposición ponderada de los mapas individuales. El mapa de multifuncionalidad del área metropolitana de Sevilla servirá de base para valorar y discutir la contribución potencial de los diferentes componentes del territorio metropolitano a una infraestructura verde multifuncional.

\section{El área metropolitana de Sevilla: un territorio diverso y complejo}

El área metropolitana de Sevilla se despliega sobre una superficie de aproximadamente $5.750 \mathrm{~km}^{2}$ y abarca un total de 51 municipios. Con una población según el censo de 2011 de 1.550.636 habitantes, constituye por tamaño la cuarta área metropolitana de España, tras las de Madrid, Barcelona y Valencia. Para su delimitación se han aplicado una serie de criterios homogeneizados internacionalmente vinculados al análisis funcional (movilidad residencia-trabajo) del sistema urbano (OECD, 2012; Feria y Martínez, 2016). El ámbito resultante de esta aproximación es asimilable a la escala adoptada por la administración andaluza para la ordenación de las aglomeraciones urbanas. Se trata de un territorio extenso, de una notable complejidad interna, donde el tejido urbano se intercala con el rico y diverso paisaje rural y natural circundante; rasgos que aportan, en su conjunto, un más que significativo potencial para la ordenación de una infraestructura verde multifuncional.

Figura 1. El área metropolitana de Sevilla. Localización y división administrativa

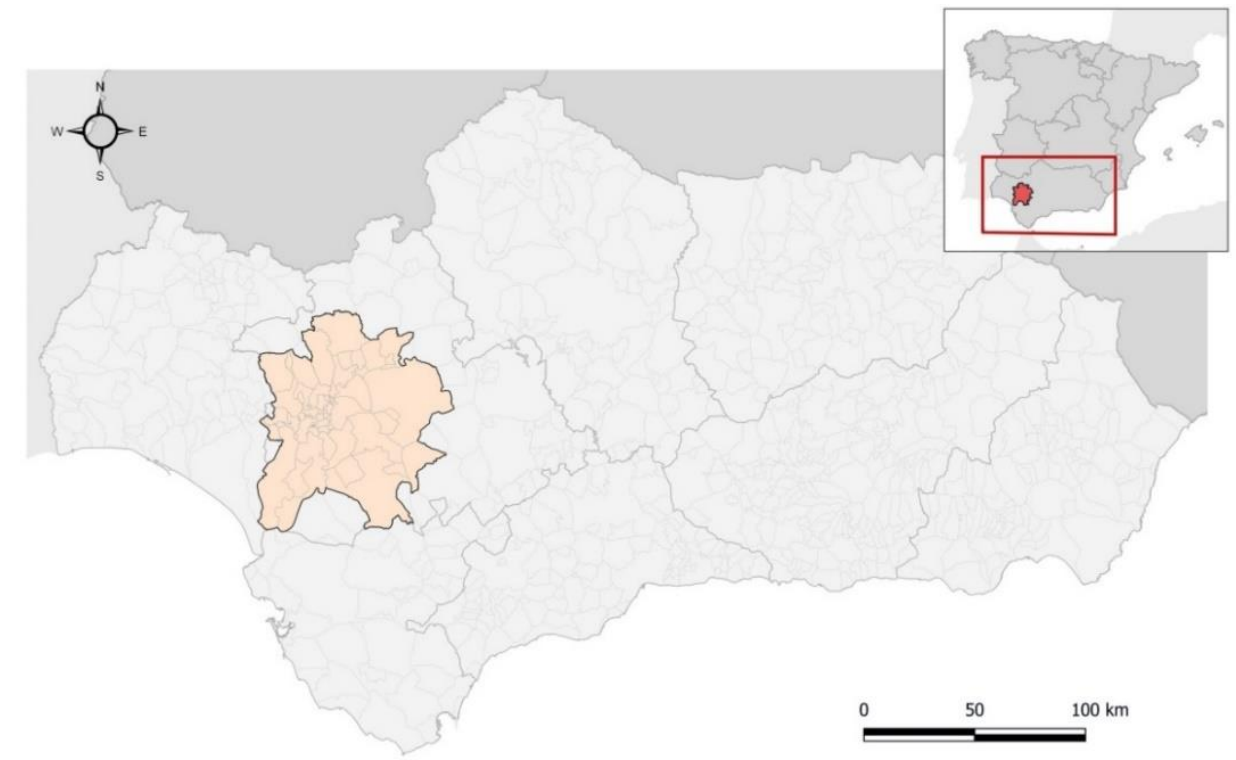

Fuente: Elaboración propia

ACE, 16 (4.6) CC BY-ND 3.0 ES | UPC Barcelona, España | Análisis de servicios ecosistémicos para la configuración de una infraestructura verde en el área metropolitana de Sevilla. DOI: http://dx.doi.org/10.5821/ace.16.46.9884 
En relación con los objetivos del presente trabajo, cabe enfatizar dos rasgos fundamentales del territorio metropolitano. El primero de ellos es su importante diversidad estructural. Tanto desde el punto de vista del medio físico como desde el de su humanización, pueden distinguirse en el territorio metropolitano cuatro sectores o ámbitos claramente diferenciados (Ojeda et al., 1998). El sector central corresponde al valle del Guadalquivir, constituido por la llanura de inundación del río y sus terrazas bajas; se trata de un ámbito de especial valor agrícola, eje histórico de comunicaciones de toda la región y en el que se localizan la ciudad central y la red de asentamientos tradicional más potente del área. Por otro lado, en el sector occidental se sitúa el Aljarafe, una plataforma miocénica elevada un centenar de metros sobre el valle, con una ocupación del suelo más fragmentada, una trama de asentamientos más densa, pero de menor tamaño y unas condiciones microclimáticas mejores que en el fondo del valle, lo que ha favorecido su poder de atracción para los desarrollos residenciales suburbanos. En tercer lugar, el sector este-sureste presenta una morfología en cuesta que se cierra con la elevación de Los Alcores, en la cual se sitúan los asentamientos tradicionales de esta parte del ámbito y que constituye el límite oriental a los procesos de urbanización difusa del territorio. Finalmente, puede identificarse una orla exterior discontinua, constituida por tierras de campiña, de uso mayoritariamente agrícola -olivar y secano- y con una potente trama de asentamientos, que puede constituirse en el futuro como ámbito preferente de expansión urbana del área metropolitana de Sevilla.

La segunda de las características básicas del área metropolitana es la fuerte fragmentación de la organización político-administrativa del territorio. Hasta la aprobación del Plan de Ordenación de la Aglomeración Urbana de Sevilla en 2009, las dimensiones, formas e intensidades del crecimiento urbano en el ámbito han sido definidas individual y aisladamente por los municipios del área a través de sus respectivos planes municipales. Las consecuencias de ello han sido numerosos desajustes y sobredimensionamientos en el proceso de ordenación del conjunto del espacio metropolitano, sobre todo en las etapas de mayor intensidad de la actividad urbanística, lo que se ha acompañado de un importante menoscabo de valiosos recursos naturales y paisajísticos. Este hecho justificaría el interés de reforzar la protección de dichos recursos frente a los futuros procesos de expansión urbana mediante una propuesta de infraestructura verde de escala supramunicipal.

\section{Funciones vinculadas a las redes: conservación de hábitats de interés natural y uso público-recreativo}

\subsection{La conservación de hábitats de interés natural}

En los ámbitos metropolitanos, la preservación de los espacios naturales más valiosos constituye una de las funciones principales de la infraestructura verde, debido al fuerte dinamismo que adquieren en estos entornos los procesos de cambio de uso de suelo, crecimiento urbano y expansión de las infraestructuras artificiales. Aunque los hábitats metropolitanos de mayor interés para la biodiversidad pueden contar previamente con la protección de alguna figura legal, su integración en una infraestructura verde metropolitana puede mejorar sus perspectivas de conservación, al potenciarse su conectividad ecológica e incrementarse su protección frente al impacto de las actividades urbanas.

A modo de indicador para la conservación de los hábitats de interés natural, se han cuantificado las coberturas de suelo de carácter natural -tanto hábitats terrestres como zonas húmedas- presentes en el área metropolitana; asimismo, se ha estimado toda la superficie sujeta a alguna figura de protección dentro de la Red de Espacios Naturales Protegidos de Andalucía (RENPA). La finalidad de estos indicadores no es abordar la biodiversidad metropolitana en su totalidad (existen, por ejemplo, especies de interés para la conservación en entornos construidos de la aglomeración urbana) ni de 
cuantificar los denominados servicios ecosistémicos de base; el objetivo es, en cambio, identificar aquellas áreas que preservan un mayor grado de naturalidad y que por ello pueden ser consideradas como piezas estratégicas en el mosaico metropolitano para la conservación de la flora y la fauna.

Los resultados muestran una más que notable presencia de hábitats de carácter natural dentro de los límites del área metropolitana: en total 157.848 hectáreas, es decir, más de un 27\% del territorio metropolitano (ver Figura 2). En cuanto a la superficie protegida, un total de 56.036 ha de cobertura natural -más de un tercio de total- se hallan sometidas a algún tipo de protección, incluyéndose tanto los espacios protegidos derivados de la legislación nacional y regional como los Lugares de Importancia Comunitaria vinculados a la Red Natura 2000. Si bien el establecimiento de figuras de protección responde a decisiones administrativas cuyo enfoque y finalidad específica ha ido evolucionando con el tiempo, se puede considerar que en su conjunto reflejan de forma sintética el elevado valor en términos ecológicos y paisajísticos de una parte significativa del territorio metropolitano.

Figura 2. Cobertura natural (\%) y espacios naturales protegidos en el área metropolitana de Sevilla

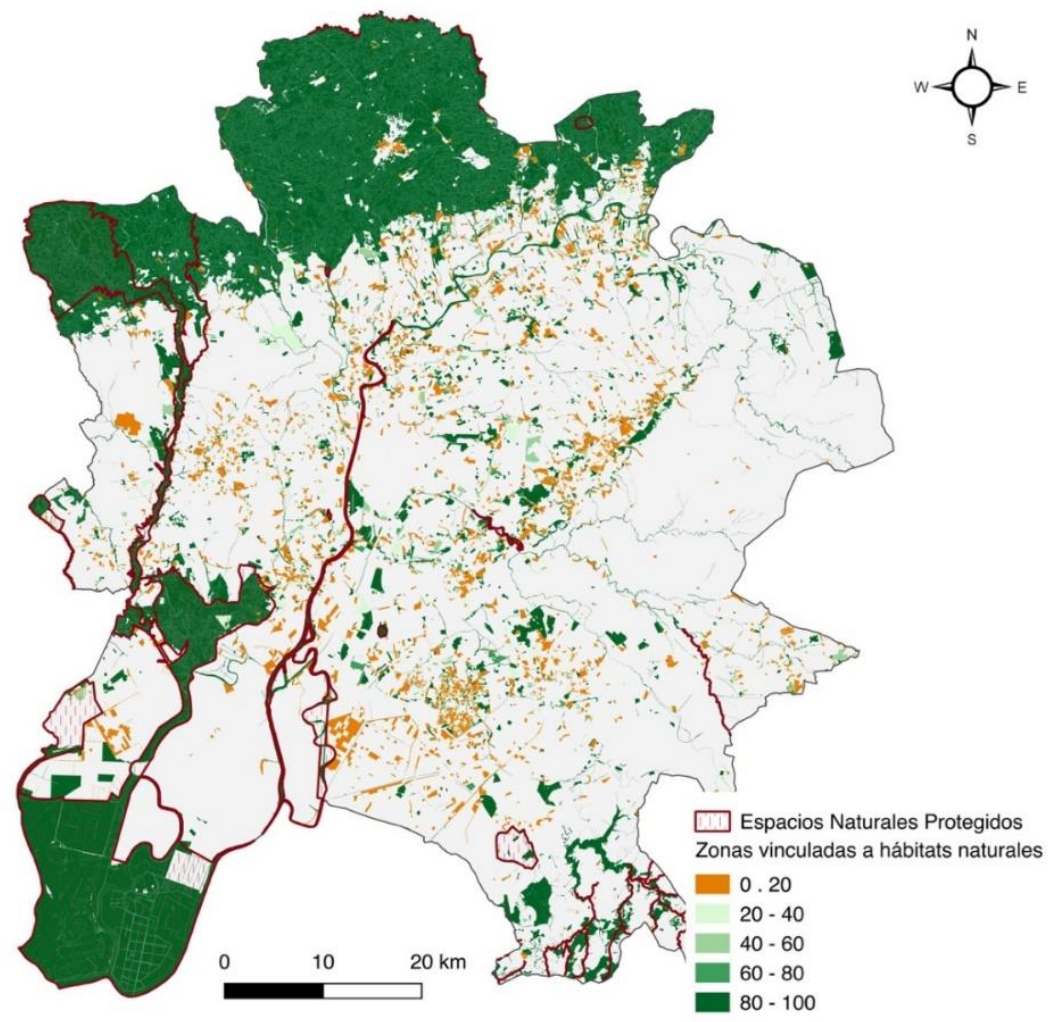

Nota: la gradación de color refleja el porcentaje de cobertura natural presente en cada polígono. Fuente: elaboración propia a partir del Sistema de Información sobre Ocupación del Suelo de España, SIOSE (Instituto Geográfico Nacional, IGN, 2011) e información de la Consejería de Agricultura, Ganadería, Pesca y Desarrollo Sostenible (CAGPDS), Junta de Andalucía

Más allá de lo anterior, la protección de las áreas naturales no debe atender sólo a su extensión superficial, sino también a su distribución y su configuración espacial. En este sentido, una de las mayores amenazas para la conservación de los hábitats en ámbitos urbanizados es el proceso de fragmentación del paisaje (AEMA, 2011b). Este fenómeno supone la división progresiva de la cobertura natural en fragmentos de menor tamaño, lo que conlleva numerosos efectos negativos para la biodiversidad: mayor aislamiento de las poblaciones de flora y fauna, restricción de los flujos

ACE, 16 (4.6) CC BY-ND 3.0 ES | UPC Barcelona, España | Análisis de servicios ecosistémicos para la configuración de una infraestructura verde en el área metropolitana de Sevilla. DOI: http://dx.doi.org/10.5821/ace.16.46.9884 
genéticos, incremento de la vulnerabilidad de los hábitats frente a los impactos y presiones externas $y$, en última instancia, pérdida progresiva de biodiversidad. El mantenimiento de la continuidad espacial de la cobertura natural metropolitana y la creación de corredores ecológicos que permitan reestablecer la conectividad perdida son criterios clave para contrarrestar el proceso de fragmentación. De ahí la importancia que tiene la configuración de la infraestructura verde como red para la provisión de esta funcionalidad.

Como se observa en la Figura 2, el área de Sevilla muestra una marcada dualidad entre la concentración de espacios naturales de notable extensión y continuidad en el sector septentrional y occidental del área, y el predominio de áreas naturales muy fragmentadas en el sector central y oriental. El diseño de una infraestructura verde metropolitana debería compensar este desequilibrio, potenciando los elementos de conexión que permitan la unión entre los fragmentos naturales más aislados y su articulación en red con los reservorios naturales más extensos y de localización más periférica. La red fluvial y otros recursos territoriales de carácter lineal -como pueden ser las vías pecuarias- ofrecen un gran potencial para este fin. A modo de ejemplo, en el sector occidental del ámbito destaca un importante conector ecológico, el corredor verde del río Guadiamar, que conecta las áreas naturales del sur -ligadas al entorno de Doñana- con los hábitats del norte -vinculados a Sierra Morena.

\subsection{Funciones de uso público-recreativo y movilidad sostenible}

El uso público es otro componente fundamental de la funcionalidad de toda infraestructura verde. A escala metropolitana, esta función queda circunscrita a ámbitos muy específicos y de dimensiones generalmente reducidas, sobre todo si se compara con otros servicios ambientales. A modo de indicador, se han cuantificado los dos tipos principales de elementos que responden a esta función en los espacios metropolitanos: los parques urbanos y los parques periurbanos. Ambas tipologías constituyen los nodos básicos de la red de espacios de uso público a escala metropolitana.

El área de Sevilla cuenta con 41 parques urbanos con una superficie total de más de 860 ha (Figura 3); cifras notables en comparación con otras aglomeraciones urbanas andaluzas, y acordes a la importante dimensión física y poblacional del ámbito. La dotación de este tipo de parques en función del número de habitantes, 5,5 ha por cada 10.000 habitantes, es también significativa. Si atendemos a los parques periurbanos, la situación es opuesta: solo existen 3 parques periurbanos, con una superficie total de 128 ha. Se trata de una cifra muy modesta en comparación con otras grandes áreas urbanas andaluzas, lo que apunta a un déficit de este tipo de espacios recreativos de carácter más extensivo y natural. Cabe matizar, no obstante, que algunas zonas protegidas y ciertos espacios informales que albergan actividades recreativas al aire libre pueden compensar, al menos parcialmente, esta carencia.

Otra faceta del uso público estrechamente ligada a la infraestructura verde es la movilidad no motorizada. Existe todo un conjunto de recursos territoriales que actúan -o pueden actuar de forma potencial- como soporte para esta función. Se han cuantificado los tres tipos más destacados: carriles bici, vías verdes y senderos, y vías pecuarias.

En lo que respecta al carril bici, Sevilla cuenta con la red más extensa de Andalucía, con un total de 294 km y 1,67 km por cada 10.000 habitantes. La red de bicicarriles tiene una clara vocación intraurbana: aunque está ampliamente desarrollada dentro de la trama urbana de la ciudad central, donde constituye una alternativa plenamente funcional de movilidad, se muestra notablemente desdibujada y falta de articulación en el entorno periurbano. En contraste, las 3 vías verdes identificadas, con una extensión de $99 \mathrm{~km}$, permiten la conexión de la ciudad con el entorno

ACE, 16 (4.6) CC BY-ND 3.0 ES | UPC Barcelona, España | Análisis de servicios ecosistémicos para la configuración de una infraestructura verde en el área metropolitana de Sevilla. DOI: http://dx.doi.org/10.5821/ace.16.46.9884 
periurbano, facilitando el acceso y disfrute del paisaje rural metropolitano por parte de los ciudadanos.

Figura 3. Parques urbanos y periurbanos y elementos conectores destinados al uso públicorecreativo en el área metropolitana de Sevilla

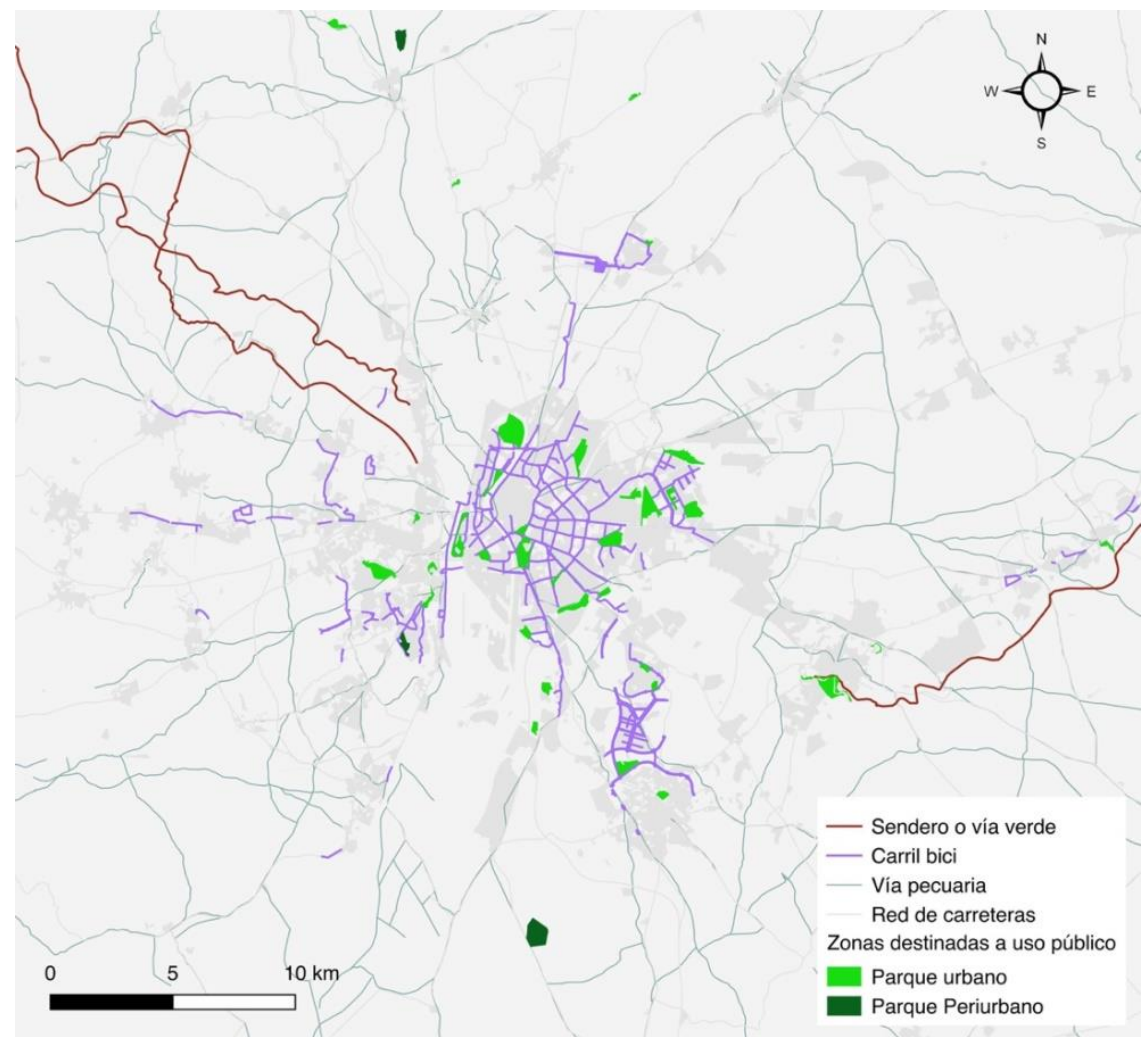

Fuente: elaboración propia a partir de los Datos Espaciales de Referencia de Andalucía, DERA (Instituto de Estadística y Cartografía de Andalucía, IECA, 2020)

Por su parte, la extensión total de vías pecuarias es mucho mayor que la de las anteriores tipologías -2.402 km y 15,19 km/10.000 habitantes-, y constituyen por su titularidad pública y su estructura en red perfectamente articulada un recurso de enorme valía para la ordenación de la infraestructura verde. Debido al desigual grado de conservación de estas vías, unido en muchos casos a problemas de ocupación del dominio público, estaríamos hablando de una funcionalidad sobre todo potencial. No obstante, parte de este viario rural ya es utilizado actualmente por la ciudadanía para pasear, hacer recorridos en bici o desplazarse de unos espacios libres a otros.

\section{Funciones asociadas a la matriz territorial: regulación ambiental y provisión}

\subsection{Funciones de regulación ambiental}

Los denominados servicios de regulación son probablemente los que tienen una mayor incidencia en la mejora de la calidad ambiental de los ámbitos metropolitanos, siendo además la tipología que representa de una forma más clara el papel multifuncional del espacio libre. La provisión de esos

ACE, 16 (4.6) CC BY-ND 3.0 ES | UPC Barcelona, España | Análisis de servicios ecosistémicos para la configuración de una infraestructura verde en el área metropolitana de Sevilla. DOI: http://dx.doi.org/10.5821/ace.16.46.9884 
servicios depende de los servicios de base y por ende está estrechamente vinculada a la preservación de la biodiversidad. No obstante, en un contexto tan artificializado como el metropolitano esta funcionalidad no solo está ligada a los ámbitos con un mayor grado de naturalidad, sino también a entornos semi-naturales y/o con una importante intervención antrópica, como el espacio agrícola. Una de las principales funciones en este sentido es la contribución a la mitigación del cambio climático, principalmente a través la captación y acumulación del carbono atmosférico por parte de la vegetación y el suelo. A modo de indicador sintético de esta función, se ha estimado que el total de carbono almacenado por la vegetación en Sevilla y su entorno es de 8.655.253 toneladas (15,04 toneladas/ha). En este sentido, el área se beneficia tanto de una significativa presencia de zonas forestales periféricas como, sobre todo, de la amplia extensión de olivar existente en el espacio metropolitano. Como se observa en la Figura 4, la conjunción de ambos factores determina que esta función se distribuya de forma bastante equitativa por gran parte de la matriz territorial.

\section{Figura 4. Nivel de almacenamiento de Carbono (C) en la vegetación del área} metropolitana de Sevilla (t/ha)

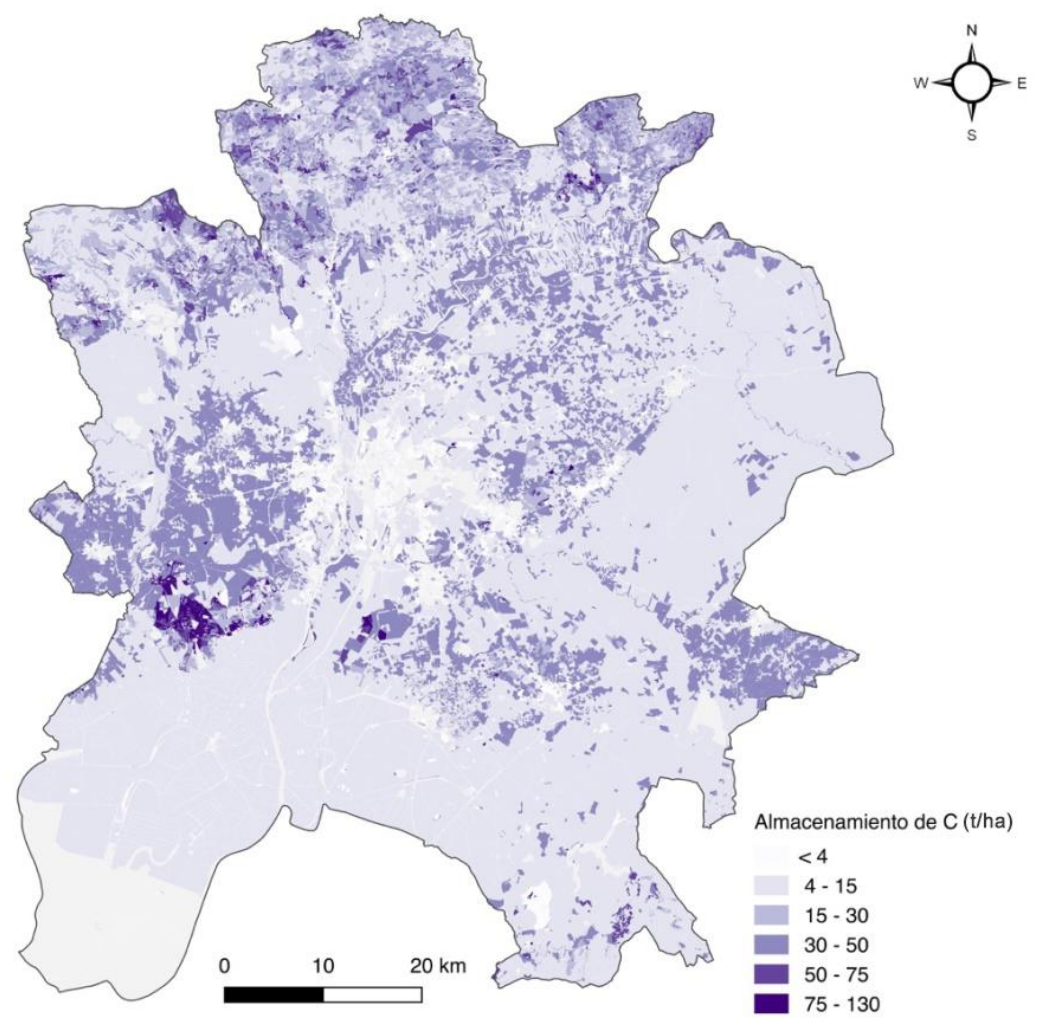

Fuente: elaboración propia a partir de SIOSE (IGN, 2011) y Gibbs (2006).

Es importante señalar que las áreas de cultivo de carácter intensivo pueden actuar no como sumideros, sino como emisores netos de gases de efecto invernadero, a consecuencia de las prácticas de manejo utilizadas y el alto consumo de energías fósiles asociado a las mismas. Por tanto, la contribución del espacio agrícola a la mitigación del cambio climático debe considerarse como una funcionalidad potencial, que depende en gran medida de las estrategias de gestión adoptadas. En este sentido, la inclusión de áreas periurbanas de cultivo como parte funcional de la infraestructura verde metropolitana puede convertirse en un factor clave para facilitar la implementación de medidas que impulsen su papel como sumideros de carbono y, al mismo tiempo, potencien otras funciones ambientales complementarias.

ACE, 16 (4.6) CC BY-ND 3.0 ES | UPC Barcelona, España | Análisis de servicios ecosistémicos para la configuración de una infraestructura verde en el área metropolitana de Sevilla. DOI: http://dx.doi.org/10.5821/ace.16.46.9884 
Otra importante función de regulación que ejerce la vegetación urbana y periurbana es la mejora de la calidad del aire, a través de la captación de compuestos contaminantes o la intercepción de partículas en suspensión. La presencia de vegetación en las ciudades tiene también un notable efecto moderador sobre la temperatura urbana, contribuyendo a mitigar la denominada isla de calor urbano gracias a la acción conjunta de la evapotranspiración y el efecto de sombra del arbolado urbano sobre edificios y superficies artificiales. Como indicador sintético para ambas funciones, se ha estimado el índice de área foliar (IAF) de las zonas ubicadas dentro de un radio de $3 \mathrm{~km}$ alrededor de las áreas urbanizadas -este umbral responde al criterio establecido por Maes et al. (2011) para el análisis de esta funcionalidad-. Para evitar la distorsión de los resultados, se han descartado en el análisis las zonas urbanas menores a 25 ha. La Figura 5 muestra la representación espacial de los resultados obtenidos. Como se puede observar, no solo es importante preservar la vegetación en el entorno del área central de la aglomeración urbana, sino también en los núcleos secundarios que constituyen la estructura polinuclear del sistema urbano. En su mayor parte, la vegetación incluida en los "anillos verdes" cartografiados no está vinculada a la presencia de parques o sistemas verdes periurbanos, sino que está ligada sobre todo a zonas de cultivos y otros espacios libres no ordenados; es decir, a la propia matriz territorial.

Figura 5. Índice de Área Foliar (IAF) en el entorno de las áreas urbanizadas

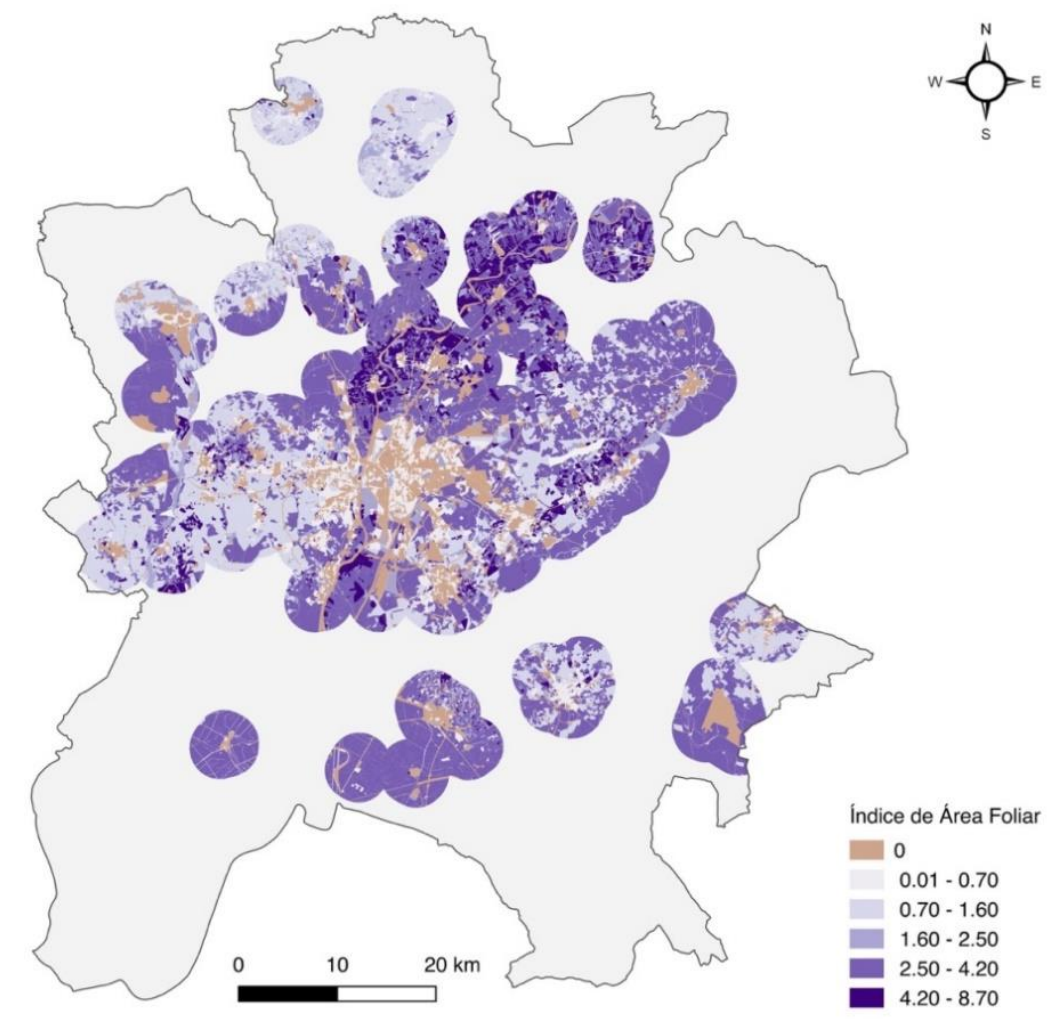

Fuente: elaboración propia a partir de SIOSE (IGN, 2011) y Santiago (2010).

Una tercera función de regulación básica para el medio ambiente metropolitano es el mantenimiento de la superficie permeable (ver Figura 6). La sustitución de la cobertura natural por superficies y estructuras de carácter impermeable modifica profundamente la circulación del agua de lluvia durante y después de la precipitación, reduciéndose tanto la capacidad de infiltración del suelo como la intercepción del agua por parte de las plantas. La consecuencia es un aumento significativo del volumen y la velocidad del agua que circula como escorrentía superficial y que acaba drenando en

ACE, 16 (4.6) CC BY-ND 3.0 ES | UPC Barcelona, España | Análisis de servicios ecosistémicos para la configuración de una infraestructura verde en el área metropolitana de Sevilla. DOI: http://dx.doi.org/10.5821/ace.16.46.9884 
el alcantarillado. Estas condiciones favorecen la denominada contaminación difusa causada por el arrastre de compuestos tóxicos, sedimentos y organismos patógenos por el agua de escorrentía. Como indicador para la función de regulación hidrológica se ha cuantificado el porcentaje de superficie permeable en el espacio metropolitano.

Figura 6. Superficie permeable (\%) por subcuencas en el área metropolitana de Sevilla
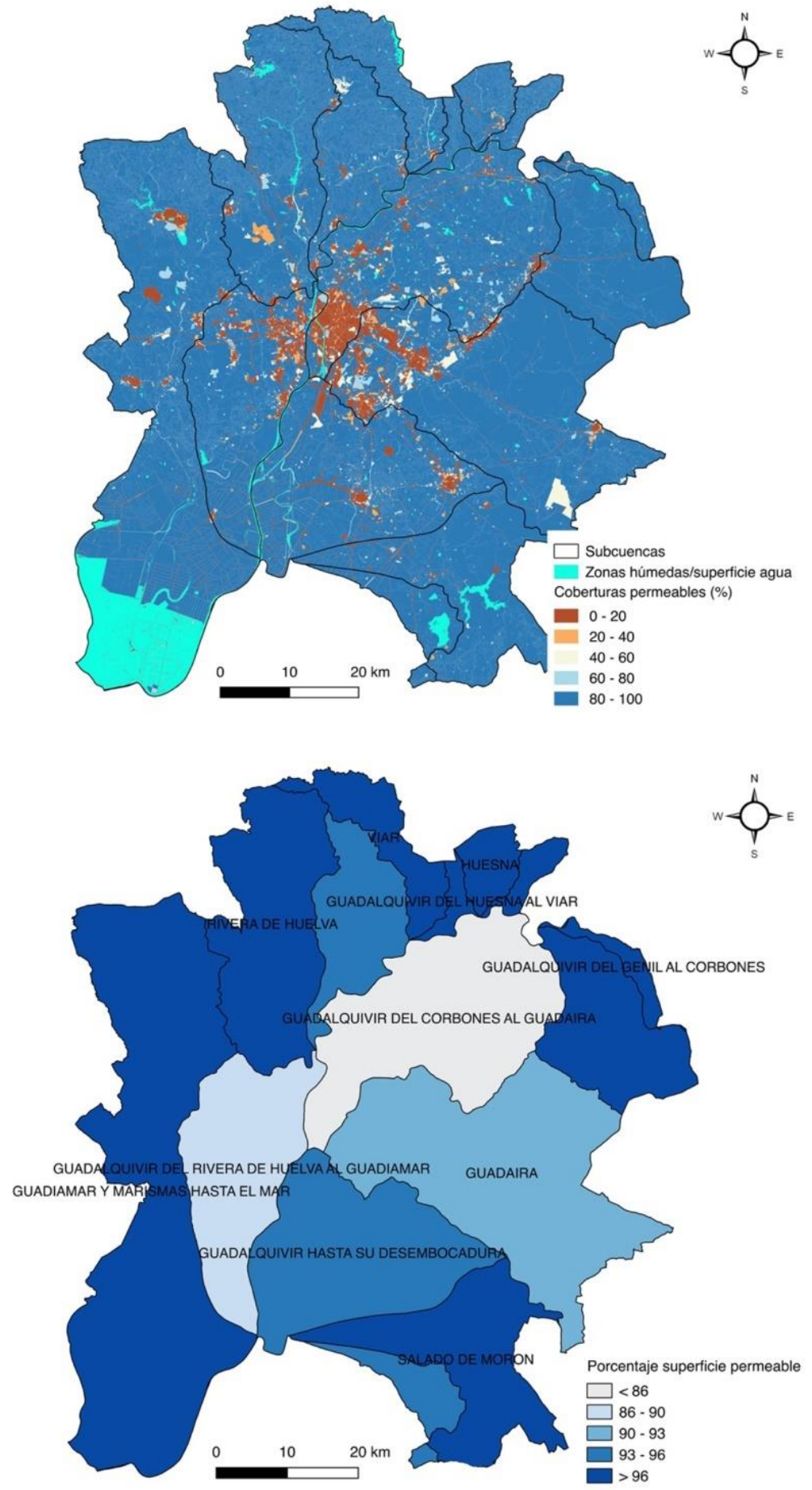

Fuente: elaboración propia a partir de SIOSE (IGN, 2011).

ACE, 16 (4.6) CC BY-ND 3.0 ES | UPC Barcelona, España | Análisis de servicios ecosistémicos para la configuración de una infraestructura verde en el área metropolitana de Sevilla. DOI: http://dx.doi.org/10.5821/ace.16.46.9884 
Diferentes estudios señalan como umbral de impacto hidrológico de referencia un 10\% de superficie impermeable a escala de cuenca (Paul y Meyer, 2008); por encima de este porcentaje, la incidencia ambiental comienza a ser significativa. En su conjunto, el área de Sevilla presenta un 7,04\% de superficie impermeable. No obstante, si se realiza el cálculo a escala de subcuenca -una subdivisión de las cuencas hidrológicas principales-, dos subcuencas metropolitanas superan el umbral del 10\%: la subcuenca denominada del "Guadalquivir, del Rivera de Huelva al Guadiamar" (13,63\% de superficie impermeable) y la denominada del "Guadalquivir, del Corbones al Guadaira" (15,07\%).

Otras subcuencas metropolitanas, también sometidas a fuertes procesos de urbanización, se encuentran cerca del umbral. En todos estos ámbitos, el diseño de la infraestructura verde debería ser tener como uno de sus objetivos prioritarios la preservación del espacio libre frente a un incremento del sellado del suelo, a fin de evitar una mayor alteración de la dinámica hidrológica.

\subsection{Funciones de provisión}

Con frecuencia, los espacios relacionados con la producción agrícola y forestal han sido los grandes olvidados en los procesos de ordenación de los ámbitos urbanos y metropolitanos, convirtiéndose a menudo en los ámbitos preferentes para la expansión de los usos urbanos. Sin embargo, en las últimas décadas se ha podido observar una progresiva reivindicación de las funciones de provisión agrícola y agroforestal prestadas por el espacio libre. Esta revaloración del espacio productivo no solo tiene como objetivo el potenciar la seguridad alimentaria de las ciudades, sino también preservar la importante contribución de la actividad agraria a la conformación del paisaje cultural y al dinamismo económico de aquellos ámbitos urbanizados donde el espacio libre posee una fuerte vocación productiva, como es habitual en el entorno mediterráneo.

En el caso de Sevilla se puede considerar que los usos agrarios constituyen la verdadera matriz del territorio metropolitano, tanto por su dominancia en términos extensión superficial -más de 366.518 ha, casi dos terceras partes del territorio metropolitano- como por su papel estructural en la configuración del espacio libre metropolitano (ver Figura 7). Los espacios cultivados periurbanos se configuran a modo de un cinturón agrario que envuelve a la ciudad metropolitana, actuando en la práctica como un equivalente de la idea de anillo verde en ausencia de otros grandes espacios abiertos menos antropizados.

La potencia económica de la actividad agrícola metropolitana, sobre todo del regadío, contribuye a explicar la resistencia de muchos de estos espacios productivos frente a la expansión de los usos residenciales, industriales o terciarios, lo que es indicativo de su papel potencial como elemento de contención de los procesos de expansión urbana. Por todo ello, no parece razonable obviar el rol potencial de la agricultura en la conformación de una infraestructura verde metropolitana verdaderamente multifuncional.

En relación con la provisión forestal (Figura 8), el área de Sevilla cuenta con 42.611 ha de masa forestal -un 7,4\% del territorio-, de las que 27.480 ha corresponderían a zonas de dehesa y 3.404 ha a plantaciones forestales. A pesar de la localización periférica de la mayoría de estos espacios, su papel para la configuración de una infraestructura verde es también muy significativo, al compaginar su dimensión productiva con un notable potencial ambiental y, en el caso de la dehesa, un fuerte carácter patrimonial ligado a su naturaleza de paisaje cultural.

ACE, 16 (4.6) CC BY-ND 3.0 ES | UPC Barcelona, España | Análisis de servicios ecosistémicos para la configuración de una infraestructura verde en el área metropolitana de Sevilla. DOI: http://dx.doi.org/10.5821/ace.16.46.9884 
Figura 7. Superficie de cultivos de secano y regadío en el área metropolitana de Sevilla

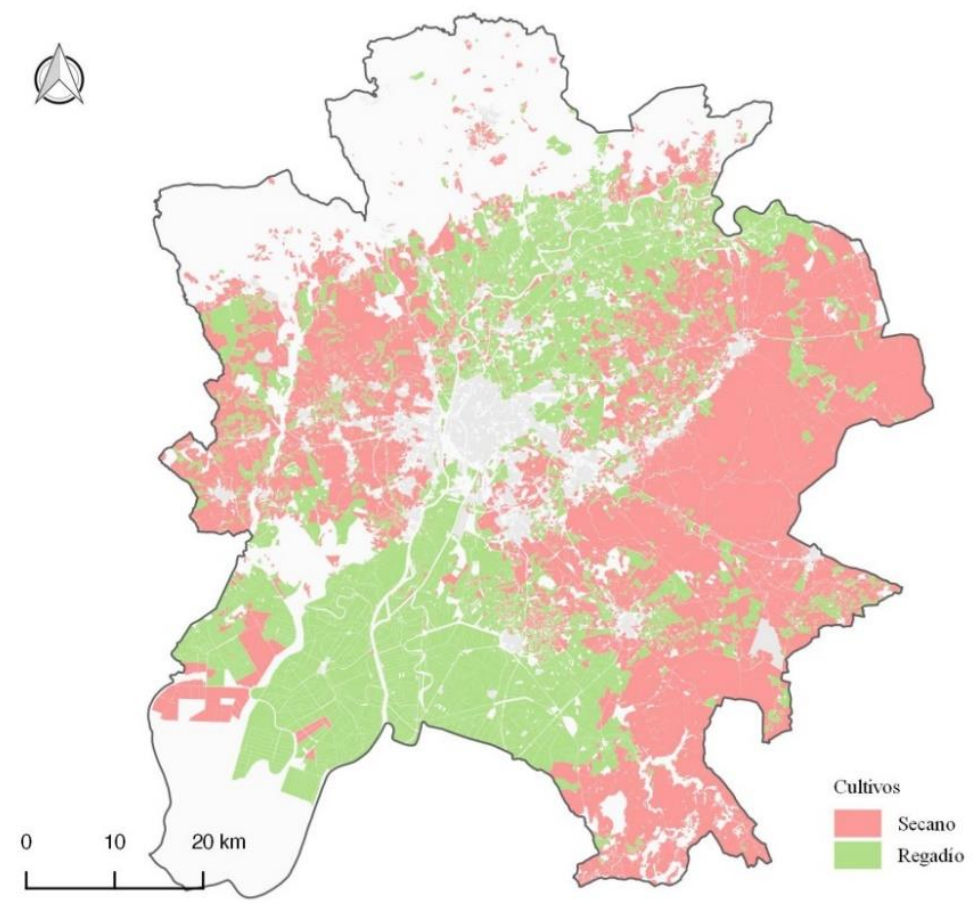

Fuente: elaboración propia a partir de SIOSE (IGN, 2011).

Figura 8. Superficie forestal (\%) y plantaciones forestales en el área metropolitana de Sevilla

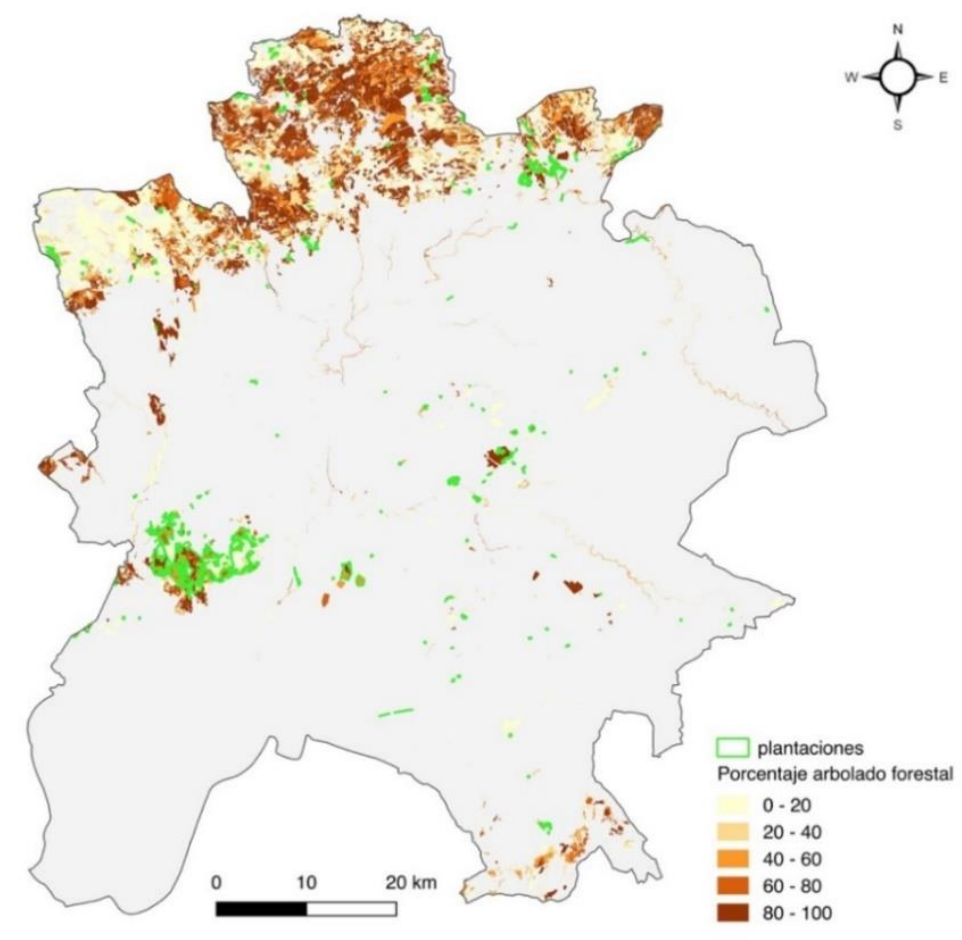

Fuente: elaboración propia a partir de SIOSE (IGN; 2011).

ACE, 16 (4.6) CC BY-ND 3.0 ES | UPC Barcelona, España | Análisis de servicios ecosistémicos para la configuración de una infraestructura verde en el área metropolitana de Sevilla. DOI: http://dx.doi.org/10.5821/ace.16.46.9884 


\section{La multifuncionalidad del territorio metropolitano y la dicotomía red - matriz territorial}

Como último paso del análisis, se han integrado en un índice único de multifuncionalidad los indicadores correspondientes a las diferentes funciones analizadas en los apartados anteriores. El proceso se ha basado en la superposición de capas ponderadas, otorgándose a cada función individual un peso específico en base a su grado de contribución a la sostenibilidad metropolitana. Para la ponderación se ha seguido un método de valoración multicriterio: el método de jerarquías analíticas (AHP) desarrollado por Saaty (1997). Esta metodología implica la jerarquización y comparación por pares de los indicadores a través de una matriz, lo que permite asignar pesos específicos a los índices en función de la importancia relativa que se les otorgue. El procedimiento tiene un carácter flexible, de forma que los pesos asignados pueden adaptarse en función de las prioridades fijadas para la infraestructura verde a partir, por ejemplo, del criterio de expertos o de la valoración social de las funciones. La Figura 9 muestra el valor normalizado obtenido para cada indicador como resultado de la metodología, asumiendo el criterio de valoración de los autores.

Figura 9. Indicadores y pesos relativos para el análisis de la multifuncionalidad

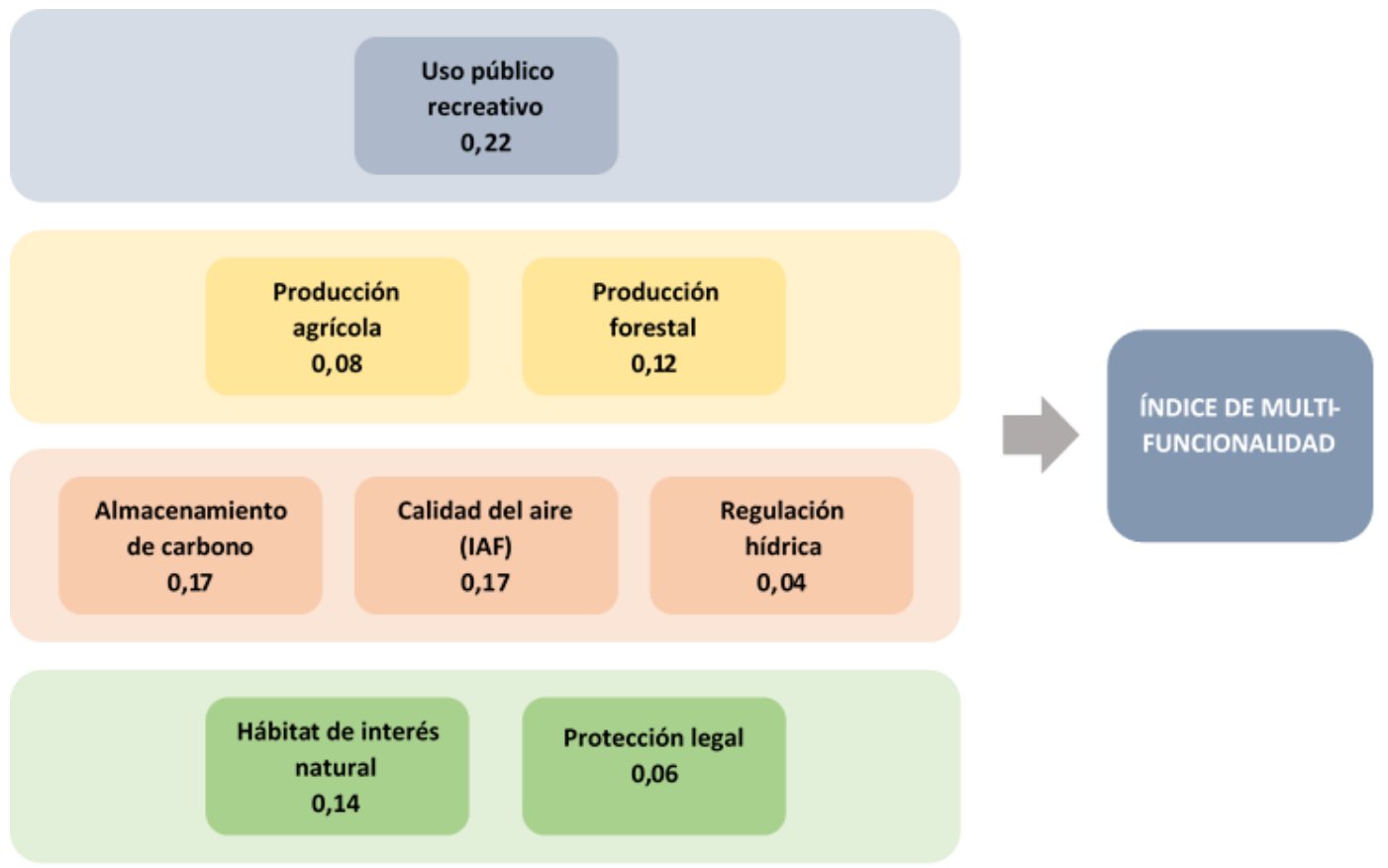

Fuente: elaboración propia.

Los valores obtenidos para cada indicador han sido normalizados en una escala de 0 a 5 y posteriormente ponderados a partir del peso asignado por el método AHP. La suma final de los valores ponderados ha permitido obtener el índice de multifuncionalidad, cuya representación cartográfica se muestra en la Figura 10.

Como se puede observar en la imagen, la distribución espacial de la provisión de servicios ligados a la infraestructura verde refleja con claridad la intrínseca complejidad estructural y funcional del territorio metropolitano. Una complejidad que invalida cualquier interpretación simplificadora sobre el espacio libre, a la vez que invita a evitar modelos preestablecidos de ordenación sin atender a la realidad local.

ACE, 16 (4.6) CC BY-ND 3.0 ES | UPC Barcelona, España | Análisis de servicios ecosistémicos para la configuración de una infraestructura verde en el área metropolitana de Sevilla. DOI: http://dx.doi.org/10.5821/ace.16.46.9884 
Figura 10. Índice de multifuncionalidad del espacio libre metropolitano

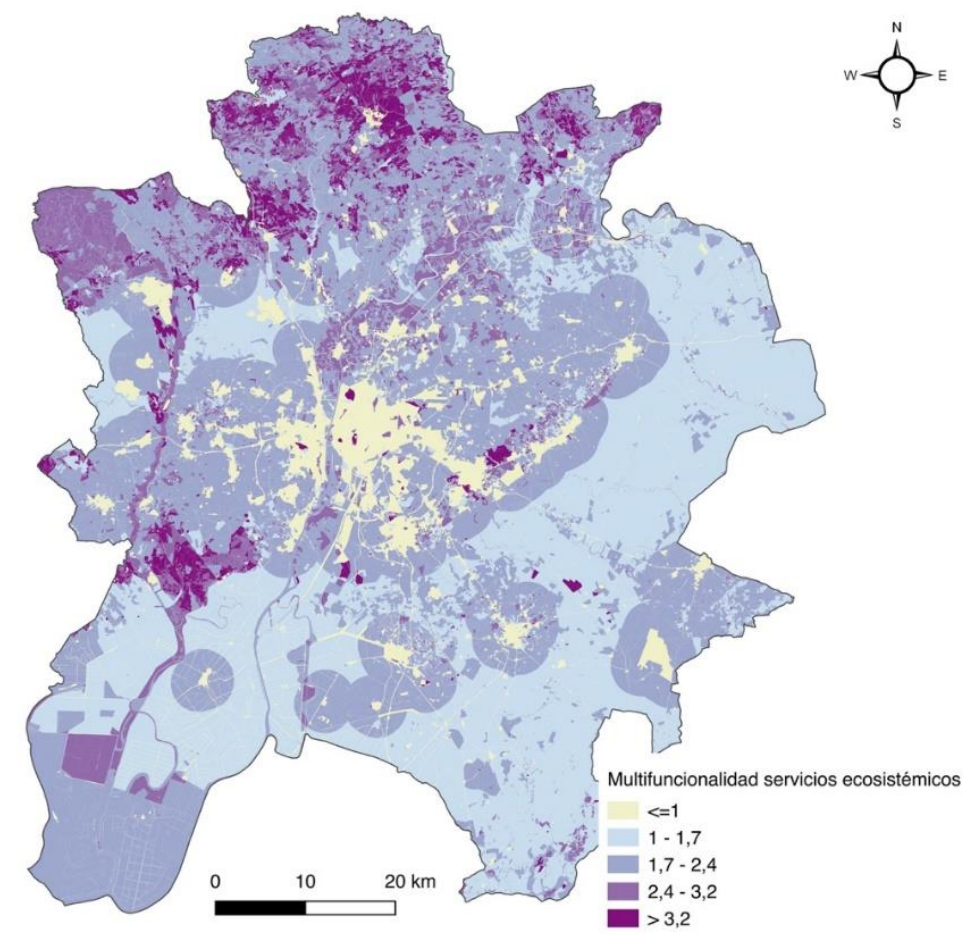

Fuente: elaboración propia

A la vista de la cartografía, es importante subrayar dos rasgos esenciales para la ordenación de una infraestructura verde metropolitana en el área de Sevilla. El primero de ellos es el papel multifuncional que ofrecen las grandes masas forestales metropolitanas -claramente destacadas en el mapa de multifuncionalidad-, al acumular funciones relativas a la conservación de la naturaleza, funciones de regulación y, en ocasiones, también funciones de provisión y de uso público. Sin embargo, su localización preeminentemente periférica hace necesario complementar la preservación de estas zonas de carácter natural con la potenciación de otros espacios libres más cercanos al núcleo central de la aglomeración urbana, de forma que se pueda incrementar la calidad ambiental de los entornos más intensamente antropizados.

El segundo rasgo es el notable papel funcional que ofrece la matriz agrícola. Como es lógico, desde un punto de vista comparativo su intensidad funcional es menor que la de las zonas que poseen un carácter más natural, pero a cambio su presencia es mucho más extensa y también más próxima a las zonas urbanas, lo que potencia su interés ambiental. Como se ha señalado, las zonas de cultivo constituyen de forma predominante la matriz estructural y funcional del paisaje periurbano, articulando el cinturón de espacios libres que rodea muchos de los principales núcleos de población, aportando funciones de regulación es este entorno próximo, y envolviendo a los espacios naturales más fragmentados.

De nuevo, es necesario realizar una matización sobre la contribución del espacio agrícola a la mejora ambiental del sistema metropolitano. atendiendo a los posibles impactos ecológicos o ecosystem dis-services (Zhang et al., 2007) potencialmente asociados a esta actividad, como la pérdida de hábitat natural, el consumo de agua para riego, la contaminación hídrica o el incremento de la erosión. Al igual que se ha indicado en el caso del balance de carbono, estos impactos dependen en gran medida de las prácticas de cultivo adoptadas, asociándose principalmente a la agricultura intensiva.

ACE, 16 (4.6) CC BY-ND 3.0 ES | UPC Barcelona, España | Análisis de servicios ecosistémicos para la configuración 16 de una infraestructura verde en el área metropolitana de Sevilla. DOI: http://dx.doi.org/10.5821/ace.16.46.9884 
Por ello, y como se ha señalado anteriormente, la inclusión de los espacios agrarios periurbanos en la planificación de la infraestructura verde puede facilitar la aplicación de medidas agroambientales que disminuyan sus efectos negativos sobre el medio y permitan un balance funcional netamente positivo.

\section{Conclusiones: planificar la infraestructura verde, más allá de la red}

Como se ha comprobado, tanto la articulación en red de parques y áreas naturales como la potenciación de la multifuncionalidad del paisaje son dos pilares esenciales para la ordenación del espacio libre metropolitano. Por un lado, la creación de redes permite reforzar el papel del espacio libre como elemento estructurante del territorio metropolitano, equiparándose así en relevancia y capacidad de organización espacial a las denominadas infraestructuras grises -infraestructuras de comunicación, energéticas, etc.-. Por otro lado, la noción de multifuncionalidad otorga al espacio libre un papel central en el funcionamiento y la dinámica del ámbito metropolitano: ya no puede ser considerado, como ha sucedido en numerosas ocasiones, un "lienzo en blanco" disponible para los nuevos desarrollos urbanos, ni tampoco el espacio residual que queda tras el proceso de urbanización. En cambio, el espacio libre se consolida como un componente clave del mosaico territorial, con un rol estratégico para la sostenibilidad de los ámbitos urbanizados.

El análisis espacial de las funciones de regulación y provisión, así como la cartografía de multifuncionalidad, apuntan a la necesidad de evitar una concepción demasiado restrictiva de la infraestructura verde, que podría derivarse de la adopción un modelo exclusivamente reticular. A la vista de los resultados, es imprescindible reivindicar el papel funcional de la matriz territorial, y en particular del espacio agrícola, como componentes esenciales de una infraestructura verde verdaderamente multifuncional. De hecho, la asunción del conjunto del espacio libre metropolitano como fuente potencial de un amplio catálogo de servicios ecosistémicos presupone avanzar, como se desprende de la cartografía aquí presentada, hacia una ordenación integral del conjunto del territorio metropolitano. Por supuesto, es necesario otorgar un peso significativo a la configuración de redes, sobre todo en relación con las funciones recreativas y de conservación de la naturaleza que se ven beneficiadas de este enfoque espacial. Pero también es importante dotar de un papel relevante $-y$ no residual o secundario, como ha sucedido con frecuencia hasta ahora- a la matriz agraria del paisaje metropolitano (Yacamán, Ferrer y Mata, 2020). Como se ha señalado, esta estrategia debe acompañarse de la adopción de medidas de gestión adecuadas que permitan minimizar los potenciales impactos negativos de las actividades agrícolas y fomentar al máximo los beneficios ambientales.

En este punto, cabe reivindicar la vigencia y la utilidad de la noción de espacio libre, como concepto maduro y plenamente asentado en la ordenación de los ámbitos metropolitanos en nuestro país. A priori, el concepto de espacio libre resulta más amplio e integrador que el de infraestructura verde, al ser capaz de englobar por su propia definición tanto la idea de red -ya sea de uso público o ecológica- como de matriz territorial. Una muestra de ello es que las iniciativas vigentes de ordenación metropolitana en España, previas a la consolidación del concepto de infraestructura verde, ya contemplan en su mayor parte la creación de redes de espacios libres y la preservación de múltiples funciones vinculadas a la matriz del paisaje (Feria y Santiago, 2017; Cruz, Oliveira y Santiago, 2017).

Por último, cabría apuntar que muchos de los retos pendientes para la planificación del espacio libre metropolitano no tienen que ver tanto con la oportunidad de adoptar nuevos desarrollos conceptuales, sino con la necesidad de avanzar en la consolidación de propuestas realmente sólidas de ordenación y dotarlas con los medios, recursos e instrumentos de gestión imprescindibles para

ACE, 16 (4.6) CC BY-ND 3.0 ES | UPC Barcelona, España | Análisis de servicios ecosistémicos para la configuración de una infraestructura verde en el área metropolitana de Sevilla. DOI: http://dx.doi.org/10.5821/ace.16.46.9884 
su efectiva materialización. En este sentido, las iniciativas de ordenación de infraestructuras verdes metropolitanas que se desarrollen en los próximos años deberían tomar como punto de partida la experiencia acumulada en materia de planificación del espacio libre a escala metropolitana.

\section{Agradecimientos}

Los autores agradecen la financiación recibida para el desarrollo de esta investigación por parte del Ministerio de Ciencia, Innovación y Universidad, a través del Programa Estatal de I+D+i (proyecto RTI2018-095325-B-IO). Asimismo, agradecen a los dos revisores anónimos del manuscrito sus comentarios y observaciones, que han contribuido a mejorar significativamente el presente trabajo.

\section{Autoría}

Jesús Santiago Ramos ha conceptualizado y diseñado la investigación, ha analizado y discutido los resultados y ha elaborado las conclusiones; Claudia Hurtado Rodríguez ha participado en el diseño metodológico, ha desarrollado el cálculo de los indicadores y ha elaborado la cartografía. Ambos autores han compartido la labor de redacción del texto.

Conflicto de intereses: Los autores declaran que no hay conflicto de intereses.

\section{Bibliografía}

Acosta, G. y Burraco, M. (1998). Glosario básico de la ordenación urbanística y territorial. Sevilla, España: Instituto Andaluz de Administración Pública.

Agencia Europea de Medio Ambiente, AEMA (2011) a. Green infrastructure and territorial cohesion. The concept of green infrastructure and its integration into policies using monitoring systems. Copenhague, Dinamarca: AEMA. Recuperado de: https://www.eea.europa.eu/publications/greeninfrastructure-and-territorial-cohesion

Agencia Europea de Medio Ambiente, AEMA (2011) b. Landscape fragmentation in Europe. Copenhague: AEMA. Recuperado de: https://www.eea.europa.eu/publications/landscape-fragmentation-in-europe

Agencia Europea de Medio Ambiente, AEMA (2014). Spatial analysis of green infrastructure in Europe. Copenhague, Dinamarca: AEMA. Recuperado de https://www.eea.europa.eu/publications/spatialanalysis-of-green-infrastructure

Alomar-Garau, G. (2021). Urbanismo crítico, cartografía y evolución del verde urbano en los planes de ensanche históricos de la ciudad de Palma (Mallorca). ACE: Architecture, City and Environment, 15(45), 9039. DOI: $\underline{\text { http://dx.doi.org/10.5821/ace.15.45.9039 }}$

Batlle, E. (2006). El sistema de espacios libres urbanos. En R. Mata y A. Tarroja (coords.). El paisaje y la gestión del territorio. Barcelona, España: Diputación de Barcelona.

Batlle, E. (2011). El jardín de la metrópoli. Del paisaje romántico al espacio libre para una ciudad sostenible. Barcelona, España: Gustavo Gili.

Benedict, M. y Mcmahon. E. (2002). Green Infrastructure: Smart Conservation for the 21st Century. Washington D.C., Estados Unidos: Sprawl Watch Clearinghouse.

Bettini, V. (1998). Elementos de ecología urbana. Madrid, España: Trotta.

ACE, 16 (4.6) CC BY-ND 3.0 ES | UPC Barcelona, España | Análisis de servicios ecosistémicos para la configuración de una infraestructura verde en el área metropolitana de Sevilla. DOI: http://dx.doi.org/10.5821/ace.16.46.9884 
Bielza, V. (2008). Introducción a la ordenación del territorio: un enfoque geográfico. Zaragoza, España: Prensa Universitarias de Zaragoza.

Bolund, P. y Hunhammar, S. (1999). Ecosystem services in urban areas. Ecological Economics, 29(2), 293-301. DOI: https://doi.org/10.1016/S0921-8009(99)00013-0

Centro de Estudios Ambientales (2014). La infraestructura verde urbana de Vitoria-Gasteiz. Documento de Propuesta. Vitoria-Gasteiz, España: CEA, Ayuntamiento de Vitoria-Gasteiz.

Comisión Europea (2013). Green Infrastructure, Gl - Enhancing Europe's Natural Capital. Communication from the Commission to the European Parliament, the Council, the European Economic and Social Committee and the Committee of the Regions. [COM, 2013) 249 final]. Bruselas, Bélgica: Comisión Europea.

Cruz, J., Oliveira, G. y Santiago, J. (2017). El espacio libre en la planificación territorial. Análisis comparado de las áreas metropolitanas en España. Ciudad y Territorio. Estudios territoriales, 193, 401416. Recuperado de https://recyt.fecyt.es/index.php/CyTET/article/view/76569

Daily, G.C., (1997). What are ecosystem services? En: Daily, G.C. (ed.) Nature's Services: Societal Dependence on Natural Ecosystems, pp. 1-10. Washington D.C., Estados Unidos: Island Press.

De Groot, R. y Hein, L. (2007). Concept and valuation of landscape functions at different scales. En: Mander, Ü.; Wiggering, H. y Helming, K. (eds.) Multifunctional land use. Meeting future demands for landscape goods and services, pp. 15-36. Berlín, Alemania: Springer.

Fariña, J. y Naredo, J. M. (2010). Libro Blanco de la Sostenibilidad en el Planeamiento Urbanístico Español.. Madrid, España: Ministerio de Vivienda.

Farrugia, S., Hudson, M.D. y McCulloch, L. (2013). An evaluation of flood control and urban cooling ecosystem services delivered by urban green infrastructure. International Journal of Biodiversity Science, Ecosystem Services y Management. volume 9(2), 136-145. DOI: https://doi.org/10.1080/21513732.2013.782342

Feria, J. M. y Santiago, J. (2009). Funciones ecológicas del espacio libre y planificación territorial en ámbitos metropolitanos: perspectivas teóricas y experiencias recientes en el contexto español. Scripta Nova, 13, 299. Recuperado de https://revistes.ub.edu/index.php/ScriptaNova/article/view/1605

Feria, J. M. (2004). Problemas de definición de las áreas metropolitanas en España. Boletín de la Asociación de Geógrafos Españoles, 38, 85-100. Recuperado de https://bage.agegeografia.es/ojs/index.php/bage/article/view/483

Feria, J.M. y Martínez, L. (2016). La definición y delimitación del sistema metropolitano español: permanencias y cambios entre 2001 y 2011. Ciudad y Territorio. Estudios Territoriales, 48(187), 9-24. Recuperado de https://recyt.fecyt.es/index.php/CyTET/article/view/76461

Feria, J.M. y Santiago, J. (2017). Naturaleza y ciudad. Perspectivas para la ordenación de la infraestructura verde en los planes territoriales metropolitanos en España. Boletín de la Asociación de Geógrafos Españoles, 74, 117-141. DOI: https://doi.org/10.21138/bage.2447

Folch, R. (2003). El territorio como sistema. Conceptos y herramientas de ordenación. Barcelona, España: Diputación de Barcelona.

Forman, R. T. T. (2008). Urban Regions. Ecology and Planning Beyond the City. Nueva York, Estados Unidos: Cambridge University Press.

ACE, 16 (4.6) CC BY-ND 3.0 ES | UPC Barcelona, España | Análisis de servicios ecosistémicos para la configuración de una infraestructura verde en el área metropolitana de Sevilla. DOI: http://dx.doi.org/10.5821/ace.16.46.9884 
García, F.M. (2019). Los valores de compacidad y densidad en los tejidos residenciales de la periferia de las ciudades intermedias españolas a lo largo del siglo XX. ACE: Architecture, City and Environment, 14(40), 11-34. DOI: https://doi.org/10.5821/ace.14.40.5690

Gibbs, H. K. (2006). Olson's Major World Ecosystem Complexes Ranked by Carbon in Live Vegetation: An Updated Database Using the GLC2000 Land Cover Product, NDP-017b. DOI: https:// doi.org/10.3334/CDIAC/LUE.NDP017.2006 (Consultado: 18/10/2019).

Hall, P. (1998). Cities in Civilization: Culture, Technology, and Urban Order. Nueva York, Estados Unidos: Pantheon Books.

Hassan, R.; Scholes, R. y Ash, N. (eds.) (2005). Ecosystems and human well-being: Current state and trends: Findings of the Condition and Trends Working Group. Washington D.C., Estados Unidos: Island Press.

Lewellyn, C., Lyons, C.E., Traver, R.G. y Wadzuk, B.M. (2016). Evaluation of seasonal and large storm runoff volume capture of an infiltration green infrastructure system. Journal of Hydrologic Engineering, 21, 1. DOI: https://doi.org/10.1061/(ASCE)HE.1943-5584.0001257

Maes, J., Paracchini, M.L. y Zulian, G. (2011). A European assessment of the provision of ecosystem services. Towards an atlas of ecosystem services. Luxemburgo, Luxemburgo: Publications Office of the European Union. Recuperado de: https://publications.jrc.ec.europa.eu/repository/handle/JRC63505

Magoni, M. y Colucci, A. (2015). Protection of Peri-Urban Open Spaces and Food- System Strategies. The Case of Parco delle Risaie in Milan. Planning Practice and Research, 32(1), 1-15. DOI: https://doi.org/10.1080/02697459.2015.1028251

Manes, F. et al. (2014). Urban ecosystem services: Tree diversity and stability of $\mathrm{PM}_{10}$ removal in the metropolitan area of Rome. Annali di Botanica, 4, 19-26. DOI: https://doi.org/10.1890/11-0561.1

Mata, R. y J. Olcina (2010). El sistema de espacios libres. En L. Galiana y J. Vinuesa (Coords.). Teoría y Práctica de la Ordenación del Territorio, 87-127. Madrid, España: Síntesis.

Momm-Schult, S.I., Piper, J., Denaldi, R., Freitas, S.R., Fonseca, M.L.P. y Oliveira, V.E. (2013). Integration of urban and environmental policies in the metropolitan area of Sao Paulo and in Greater London: the value of establishing and protecting green open spaces. International Journal of Urban Sustainable Development, 5, 89-104. DOI: https://doi.org/10.1080/19463138.2013.777671

Ojeda, J. et al. (1998). El Área Metropolitana de Sevilla. Análisis del medio físico. Sevilla: Junta de Andalucía. https://www.juntadeandalucia.es/medioambiente/portal web/servicios generales/doc tecnicos/199 8/area metropolitana sevilla/area metropolitana sevilla analisis.pdf

Organización para la Cooperación y el Desarrollo Económicos, OECD (2012). Redefining Urban. A new way to Measure Metropolitan Areas. París, Francia: OECD. Recuperado de: https://www.oecd.org/regional/redefiningurbananewwaytomeasuremetropolitanareas.htm

Paul, J. P. y Meyer, J. L. (2008). Streams in the urban landscape. En Marzluff, J. M., et al. (eds.) Urban Ecology. An International Perspective on the Interaction Between Humans and Nature. Nueva York, Estados Unidos: Springer, 207-231.

Pedrazzini, L. (coord.) (2011). Ámbitos periurbanos. Líneas guía paisajísticas para la gobernanza del territorio. Monza, Italia: Regione Lombardia - D.G. Sistemas Verdes y Paisaje. 
Rodríguez, V. y Aguilera, F. (2016). ¿Infraestructuras verdes en la planificación española? Ciudad y Territorio. Estudios Territoriales, 189, 399-418. Recuperado de: https://recyt.fecyt.es/index.php/CyTET/article/view/76490

Rodríguez-Avial, L. (1982). Zonas verdes y espacios libres en la ciudad. Madrid, España: Instituto de Estudios de la Administración Local.

Rueda, S. (2012). Libro Verde de la Sostenibilidad Urbana y Local en la Era de la Información. Madrid, España: Ministerio de Agricultura, Pesca, Alimentación y Medio Ambiente. Recuperado de: https://www.mitma.gob.es/areas-de-actividad/arquitectura-vivienda-y-suelo/urbanismo-y-politicade-suelo/urbanismo-y-sostenibilidad-urbana/libro-verde-de-sostenibilidad-urbana-y-local-en-laera-de-la-informacion

Saaty, T.L. (1997). On the Measurement of Preferences in the Analytic Hierarchy Process: Discussion. Journal of Multicriteria Decision Analysis, 6, 324-324. DOI: https://doi.org/10.1002/(SICl)10991360(199711)6:6<309::AID-MCDA163>3.0.CO;2-2

Santiago, J. (2008). La Naturaleza en la Ciudad. Perspectivas teóricas y metodológicas para el estudio de la funcionalidad ambiental del espacio libre. Sevilla: Consejería de Obras Públicas y Transportes, Junta de Andalucía. Recuperado de: https://ws147.juntadeandalucia.es/obraspublicasyvivienda/publicaciones/07\%20PAISAJE\%20Y\%20TE RRITORIO/la naturaleza en la ciudad/la naturaleza en la ciudad.pdf

Santiago, J. (2010). Ciudad, espacio libre y funcionalidad ecológica. Una aproximación territorial al estudio del medio ambiente en el área metropolitana de Sevilla (Tesis doctoral). Sevilla, España: Universidad Pablo de Olavide.

Tzoulas et al. (2007). Promoting ecosystem and human health in urban areas using green infrastructure: a literature review. Landscape and Urban Planning, 81, 167-178. DOi: https://doi.org/10.1016/j.landurbplan.2007.02.001

Vera, F., Olcina, J., y Sainz, A. (2019). La incorporación de la infraestructura verde en la Ordenación Territorial. El Plan de Acción Territorial de la Infraestructura Verde del litoral de la Comunidad Valenciana, PATIVEL. Ciudad Y Territorio. Estudios Territoriales, 201, 467-490. Recuperado de: https://recyt.fecyt.es/index.php/CyTET/article/view/77538

Yacamán, C., Ferrer, D., y Mata, R. (2020). Green Infrastructure Planning in Metropolitan Regions to Improve the Connectivity of Agricultural Landscapes and Food Security. Land, 9(11), 414. DOI: https://doi.org/10.3390/land9110414

Zhang, W.; Ricketts, T.; Kremen, C. y Carney, K.M. (2007) Ecosystem services and dis-services to agriculture. Ecological Economics, 64, 253-260. DOI: https://doi.org/10.1016/j.ecolecon.2007.02.024 